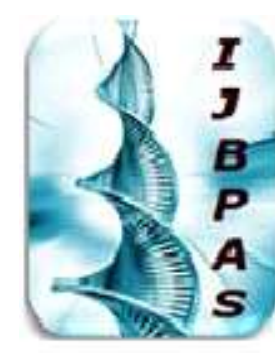

International Journal of Biology, Pharmacy and Allied Seiences (IJBPAS) 'A Bridge Betuen Caboratory and Qnendo'

WWw.ijbpas.com

\title{
A REVIEW ON NOVEL INSIGHTS OF PELLETIZATION AND SPHERONIZATION TECHNIQUES
}

\section{NAYKODI VK*, SONAWANE AA, DHOBALE SM, JADHAV SL AND GAIKWAD DD}

Vishal Junnar Seva Mandal's Vishal Institute of Pharmaceutical Education and Research, Ale, Tal. Junnar, Dist. Pune, M.S., India

*Corresponding Author: E Mail: naykodivrushali111@gmail.com

Received 27 ${ }^{\text {th }}$ April 2021; Revised $25^{\text {th }}$ June 2021; Accepted $1^{\text {st }}$ Aug. 2021; Available online $1^{\text {st }}$ Oct. 2021

https://doi.org/10.31032/IJBPAS/2021/10.10.1041

\section{ABSTRACT}

The review article deals with the extrusion and spheronization (pelletizer) techniques utilized for spheroidal particle formation in the pharmaceutical industry i.e., pellet for mainly oral administration which can be further formulated into several other dosage forms such as tablets, capsules or can be administered as such. Now-a-days oral administration has become the most versatile, convenient and common route of drug administration which ultimately focuses on patient compliance. The technique which is setting horizon in pelletization is "Extrusion Spheronization" because of its simple and easy steps involved in pellet production in a faster way. This review also includes the characterization and evaluation of pellets to ensure its quality, safety and efficacy to give out the required therapeutic activity after administration.

Keywords: Pelletizer, Extruder and Spheronizer, Pellets, Pelletization

\section{INTRODUCTION}

\section{Pellets:-}

In the pharmaceutical industry, pellets are compact ,dense ,uniform size simple small free-flowing spherical particles formed by agglomeration of fine powder or granule pellets are mainly used in formulation and development of control release dosage form. Pellets can be given in the form of disintegrating tablets, suspensions and capsules showing number of advantages over 
the single unit dosage form [1]. The size range of pellet size is of $0.5-1.5 \mathrm{~mm}$, and are intended usually for oral administration [2]. Pellets have the free flowing capacity and have low porosity about $10 \%$. At present usage of pellets has increased largely due to their advantages and there novel approaches, the novel approaches of pellets includes;

1) They help in preparation of modified release multiple dosage form with different release patterns like immediate and sustained release pattern,

2) They help in taste masking of the drugs which are bitter in taste,

3) They are available as mouth melt pellets,

4) Polymer based pellets for control release pattern of drug,

5) As fast dissolving tablets containing micro pellets,

6) As a self-emulsifying pellets,

7) Gastro retentive floating pellets etc.

Thus, the usage of pellets provides novel approaches to the patients in providing accurate, and easy in administrating the dosage form [3-4].

\section{Advantage of Pellets :-}

Pellets offer a significant number of advantages over conventional unit-dosage form [5-9].

\section{Technological Advantages:-}

1. Uniformity of dose. Layering techniques and extrusionspheronization offers great accurancy with uniform drug delivery to the pellets.

2. Spheres have excellent flow properties. This becomes very useful in automated processes or in processes where exact dosing is required, e.g. tableting, moulding operations, capsule filling and packaging.

3. Prevention of dust formation, resulting in an improvement of the process safety, as fine powder can cause dust explosions and the respiration of fines can cause health problems.

4. Controlled release application of pellets due to the ideal low surface area-to-volume ratio that provides an ideal shape for the application of film coatings.

5. They can be blended to deliver incompatible bioactive agents simultaneously and to different release profiles at the same or different sites in the gastrointestinal (GI) tract. 
6. Extrusion Spheronization technique gives uniformity of dose with excellent accuracy.

\section{Therapeutic Advantages:-}

7. Pellets can disperse freely throughout the GIT after administration and consequently the drug absorption is maximized.

8. The wide distribution of spherical particles in the gastrointestinal(GI) tract limits localized build-up of the drug, avoiding the irritant effect of some drugs on the gastric mucosa

9. Reduce inter and intra patient variability.

10. It improves safety and efficacy of a drug.

11. Less susceptible dose dumping.

\section{Disadvantages:-}

1. Dosing by volume rather than the number and splitting into single dose units as required.

2. Involves capsule filling which can increase the costs or tab letting which destroy film coating on pellets.

3. Filling of pellets in a capsule is expensive.

4. Production process is very difficult to control, since several critical process parameters such as the amount of water needed, time, pressure, etc.[10]
Desirable Properties of Pellets:- [11]

1. For Uncoated pellets

a. Uniform spherical size

b. Narrow particle size distribution

c. Good flow property

d. Low friability

e. Even surface

f. Low dust formation

g. Reproducible packing

h. Ease of coating

\section{For Coated pellets}

a. Maintain all above properties

b. Desirable drug release characteristics

Formation and growth mechanism of pellets

In order to select and optimize any pelletization process, it is essential to understand the fundamental mechanisms of pellet formation and growth. Nucleation, coalescence, layering, abrasion transfer and size reduction are the events that lead to the formation and growth of pellets. In nucleation, primary particles are drawn together to form three-phase air-water-solid nuclei (Fig. 1A). The collision of wellformed nuclei to form larger size particles is known as coalescence (Fig. 1B). Successive addition of material on already formed nuclei is layering (Fig. 1C). Transfer of material from one particle to another without any preference in either direction is abrasion 
transfer (Fig. 1D). There are three size reduction mechanisms which have an indirect effect on the growth mechanism, particularly layering and to some extent coalescence. Well-formed particles may undergo size reduction due to attrition (Fig. 1E), breakage (Fig. 1F) and shatter (Fig.G) [12].

A) Nucleation:-Nucleation is common stage in all pelletization process usually it arises when the powder is wetted with binder solution. Here the primary particles are strained together to form three phases that is air-waterliquid nuclei these nuclei are attached to one another by the liquid bridges which are pendular in nature. Nucleation is followed by a transition stage; here the growth mechanism affects the transition stage due to coalescence and layering.

B) Coalescence:-The coalescence is defines as well formed nuclei due to the collision of large size particles, during this step the total mass remains uncharged and the number of nuclei reduces gradually.

C) Layering:-Pellet formation by layering involves the deposition of successive layers of drug molecules from dry powder or granules, suspension, a solution of drug particles. At this point the third phase, the ball growth region, is reached.

D) Abrasion transfer:-The layering step involves the layering of already formed nuclei by sequential addition of material. Followed by transfer of material from one pellet to another without any predilection in the direction is abrasion transfer $[\mathbf{1 2}, \mathbf{1 3}]$.

\section{Pelletization Techniques:-}

Depending on the type of equipment and processes selected, pellet formation and growth may occur in a number of ways (Fig. 2). Here are phenomena that describe the systematic for-mation of pellets during the various pelletization processes.

\section{Agitation:-}

In agitation, finely divided particles are converted to spherical particles, upon the addition of appropriate quantities of liquid, by a continuous rolling or tumbling motion. The liquid may be added prior to or during the agitation stage. Pans, discs, drums, or mixers may be used to produce pellets by the balling process [14].

\section{Compaction:-}

A compaction is a form of pressure agglomeration in which drug particles or granules are forced together with 
or without formulation aids by a mechanical force to generate pellets of well-defined shape and sizes. In compression, particles that are pretreated through dry blending or wet granulation followed by drying rearrange themselves to form a closely packed mass. At higher pressure, the particles are forced against each other and undergo elastic and plastic deformation. In extrusionspheronization, first the dry powder mix is agglomerated with the help of a binding liquid. Then it is processed in the extruder to produce highdensity extrudates. These extrudates are finally converted to pellets on spheronizer [15].

\section{Drug layering:-}

Pelletization by layering involves the deposition of successive layers of drug entities from solution, suspension, or dry powder on preformed nuclei, which may be

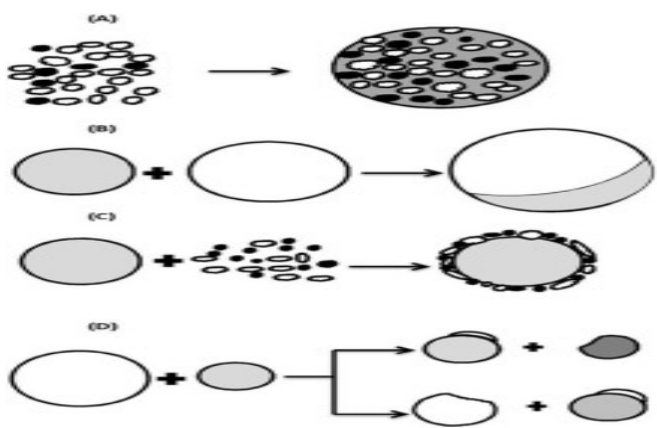

crystals or granules of thesame material or inert starter material. In powder layering, a binder solution is first sprayed onto the nuclei, followed by the addition of powder. The moist nuclei tumble in the rotating panor disc, pick up powder particles and form layers of small particles that adhere to each other and the nuclei by means of capillary forces developed in the liquid phase. As additional binding liquid is sprayed, layering of more powder on the nuclei continues until the desired pellet sizes are obtained

(Fig.3).

In solution/suspension layering, the drug particles are dissolved or suspended in the binding liquid. The liquid is then sprayed nuclei and spread out on nuclei followed by drying. Spreading depends on the droplet wetting characteristics, the wettability of the material, and droplet dynamics.

Figure 1: Formation and growth mechanism of pellets (A) nucleation, (B) coalescence, (C) layering and (D) abrasion transfer and mechanism of size reduction (E) attrition, (F)breakage and (G) shatter 


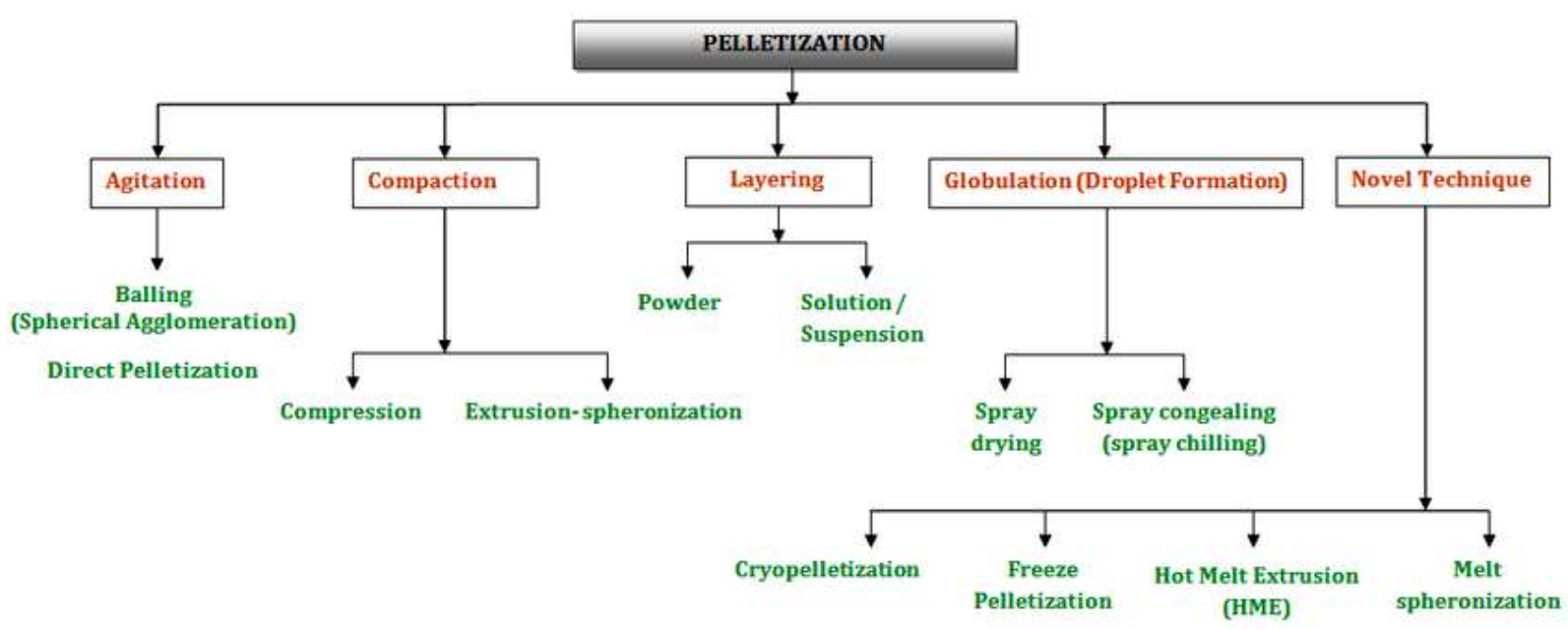

Figure 2: Classification of pelletization technique

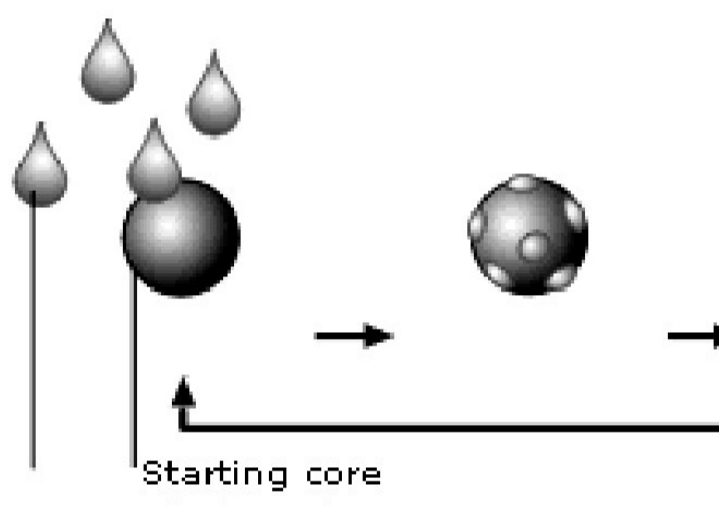

Suspended auxiliary matter and dissolved binder

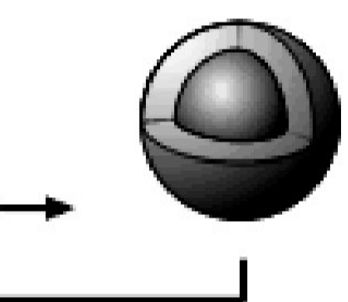

First layer

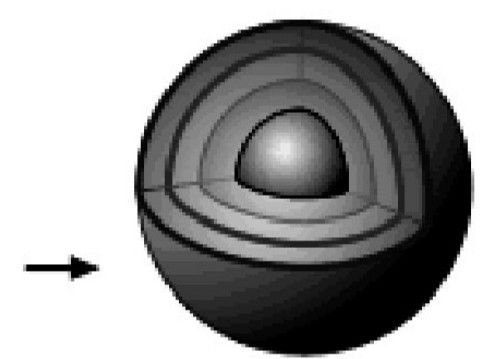

"Onion" strutture

Figure 3: Drug layering using solution
Spraving
Folling
Dryingl
Laver formation
Pellet

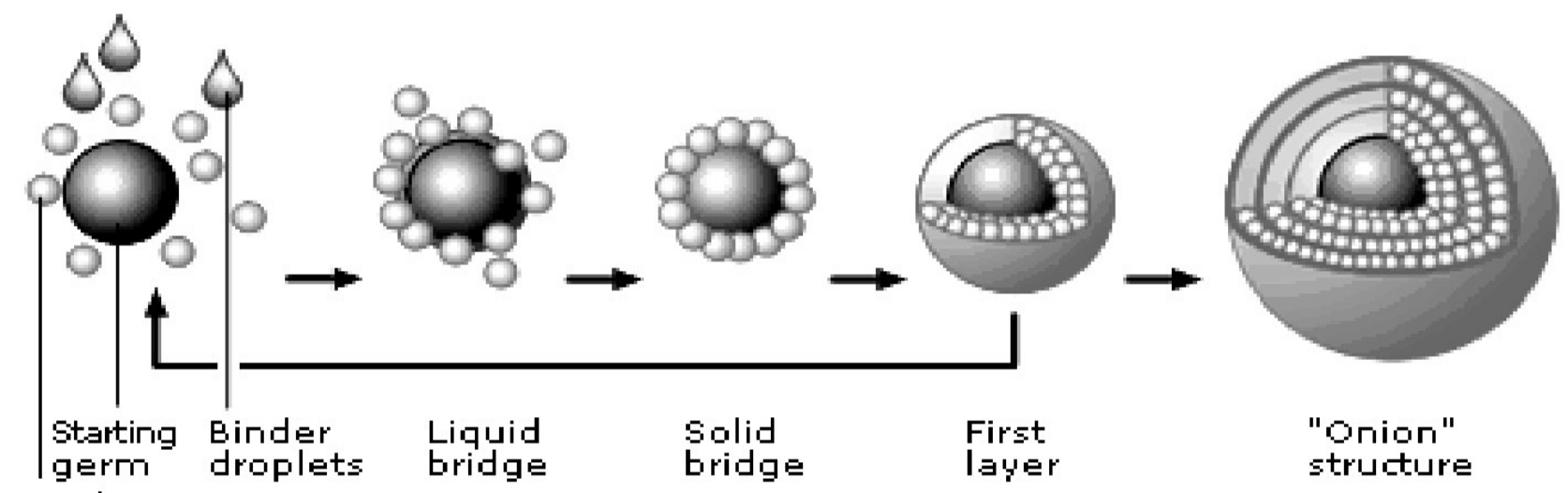

Powder

Figure 4: Drug layering By Using Suspension 


\section{Globulation:-}

Globulation is a process where liquid materials like melt, solution, or suspension are atomized to generate spherical particles or pellets. During spray drying, the atomized droplets are contacted by a hot gas stream and evaporation of the liquid is initiated. Evaporation involves simultaneous heat and mass transfer and depends on the temperature, humidity, and transport properties of the air surrounding the droplet. During spray congealing, the atomized droplets are cooled to below the melting point of the vehicles. A critical requirement in this process is that substances should have well-defined melting points or small melting zones [12-16].

Pharmaceutical co-crystals are used as a strategy to overcome poor physicochemical properties of drugs.

Duarte et al. have tried spray congealing for the first time in the preparation of co-crystals [17].

\section{Spheronization:-}

In spheronization, the extruded, cylindrically shaped particles are broken into uniform lengths and are gradually transformed into spherical shapes; this shaping process is due to plastic deformation. As extrudates are first broken into nearly uniform lengths, all three dimensions of agglomerate shape are determined, and spheres with a nearly uniform diameter are produced. In the spheronization process, different stages can be distinguished depending on the shape of the particles, i.e., starting from a cylinder over a cylinder with rounded edges, dumbbells, and elliptical particles to eventually perfect spheres (Fig. 5A). Baert and Remon suggested that another pelletforming mechanism might exist (Fig. 5B). In this mechanism, a twisting of the cylinder occurs after the formation of cylinders with rounded edges, finally resulting in the breaking of the cylinder into two distinct parts. Both parts have a round and a flat side. Due to the rotational and the frictional forces involved in the spheronization process, the edges of the flat side fold together like a flower forming the cavity observed in certain pellets [18-19].

A spheronizer is a device consisting of a vertical hallow cylinder with a horizontal rotating disk (friction plate) located inside (Fig. 6). Extrudates are charged onto the rotating plate and broken into short segments by contact with friction plate, collisions between particles and collisions with the wall. Mechanical energy introduced by the spinning friction plate is transmitted into 
kinetic energy in the form of mechanically fluidized bed. Further processing will cause the extrudate to deform gradually into a spherical shape. The friction plate has a grooved surface to increase the frictional forces. Two types of the geometry of the grooves exist, cross-hatch geometry where the grooves form right angles and radial geometry where a radial pattern is used.
Fig. 7 shows the fundamental components of the spheronizer. The most important component is the friction plate (Fig. 7) which can have a variety of surface textures designed for specific purpose. The crosshatch pattern is most common where the grooves intersect each other at $90^{\circ}$ angles.
(A)

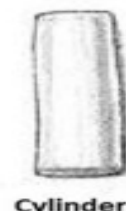

(B)

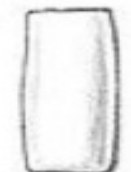

Cylinde
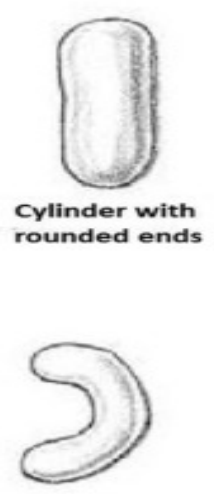

Rope

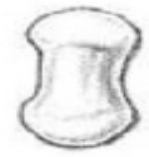

Dumbell shape

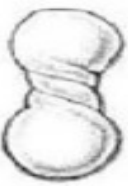

Twisted dumbell shape

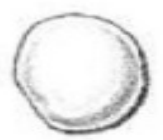

Ellipsoid

Sphere
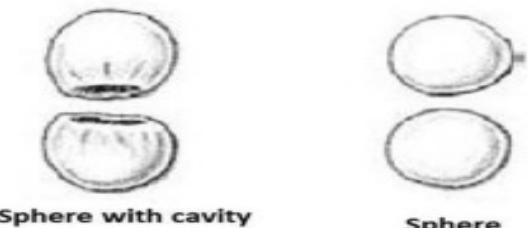

Sphere

Figure 5: Pellet-forming mechanism according to (A) Rowe and (B) Baert

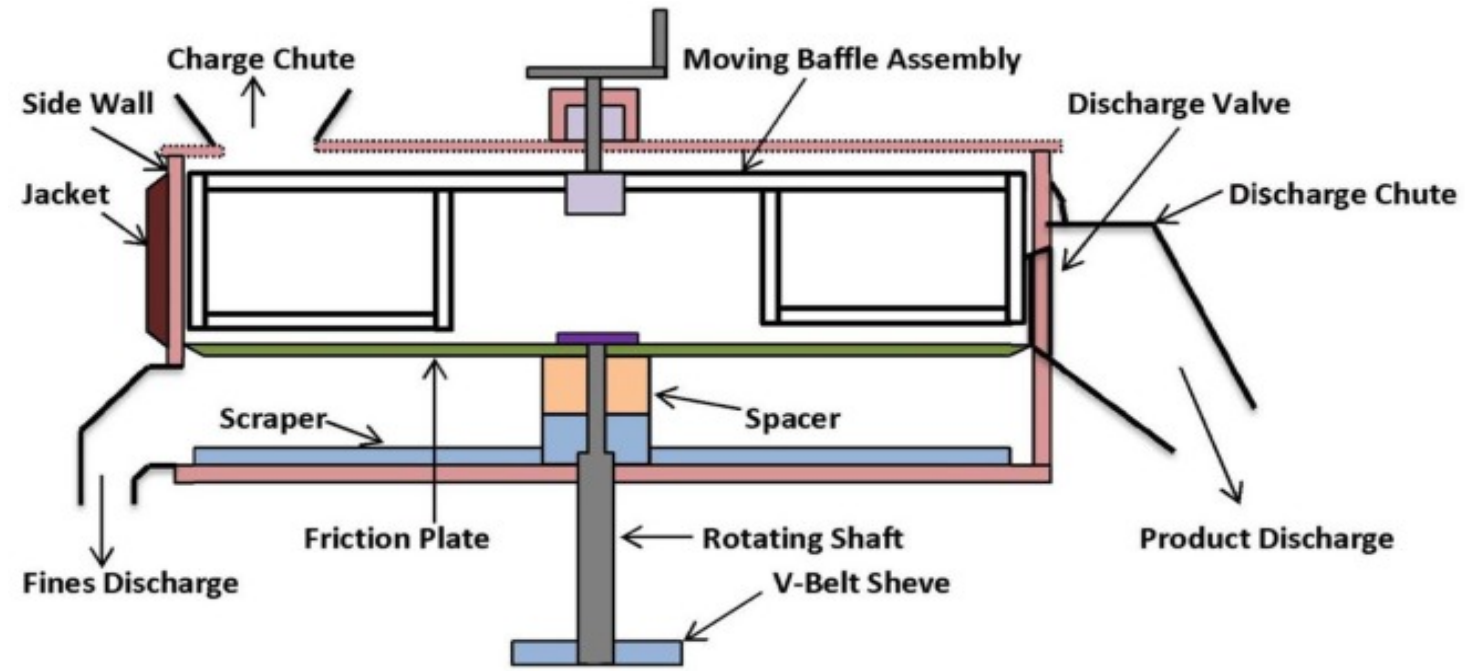

Figure 6: Schematic of spheronizer 


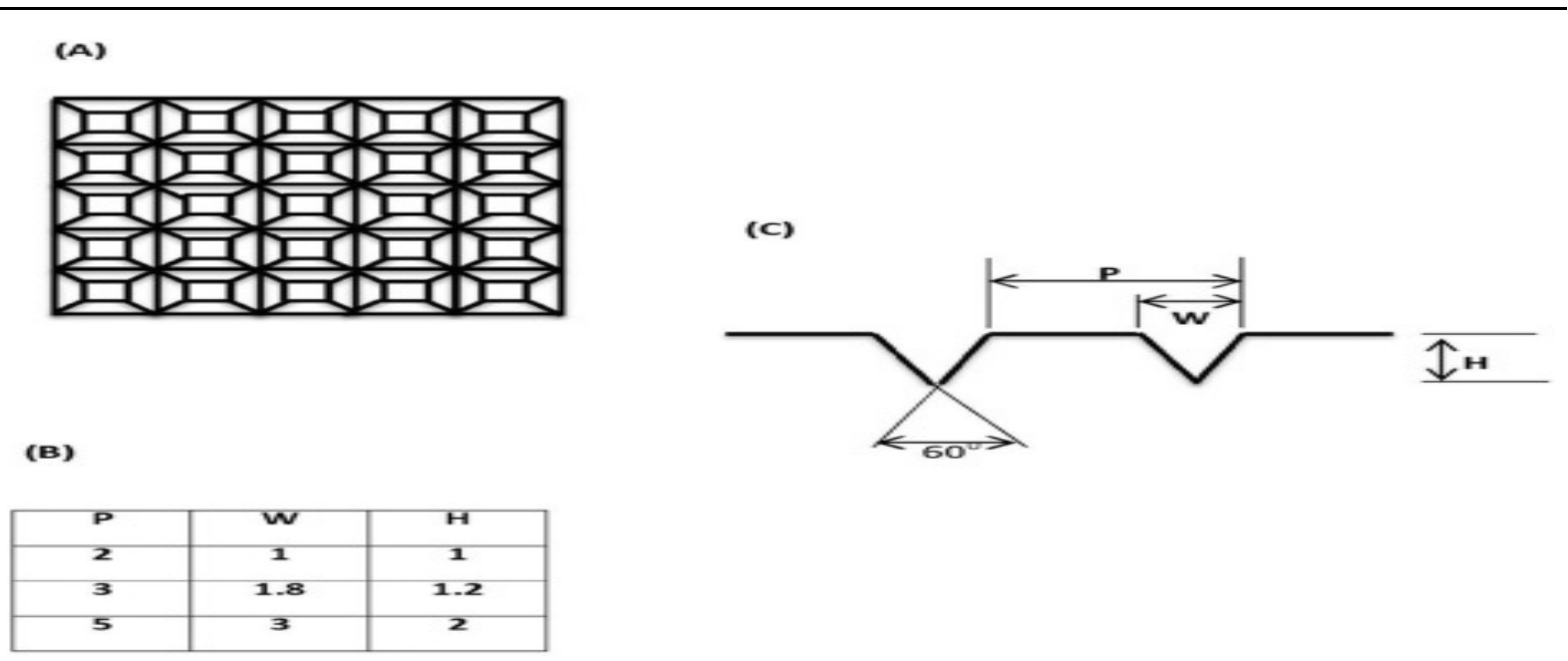

Figure 7: Typical grid pattern of friction plate: (A) detail of grid pattern (cross-hatch design); (B) typical dimensions of plate design (mm); (C) cut away view of the plate

\section{FACTORS \\ AFFECTING}

\section{PELLETIZATION TECHNIQUE}

\section{Moisture content:-}

Moisture in the wet mass brings cohesiveness to powder so that the wet mass can be extracted and spheronizer to give spherical shape. High moisture contents lead to agglomeration of pellets during the process of spheronization.

\section{Rheological characteristics:-The} optimum rheological condition leads to good flow ability in order to extrudate the wet mass. The rheological variations make improper and non-uniform extrudate.

3. Solubility of excipients and drug in granulating fluid :-

Soluble drug get dissolve in a granulating liquid. Thus increasing the volume of liquid phase leads to over wetting of pellets. But increase in wetting liquid increases plasticity but includes sticky mass.

\section{Composition of granulating fluid:-}

Besides water, alcohol, water/alcohol mixture, ethyl ether, dilute acetic acid, isopropyl alcohol is used as a granulating liquid. Aqueous polymer dispersion containing HPMC, PVP, etc. can also be used as granulating fluid.

5. Physical properties of starting material:-

Quality of pellets depend not only composition but also on different grades of the same product. The swelling property of material used in pelletization technique decides the release rate of drug in pellets.

\section{Speed of Spheronizer :-}

It affects the size, hardness, sphericity and density of pellets. The high speed gives high sphericity, lower friability, 
smooth surface and higher crushing strength.

\section{Extrusion screen:-}

The quality of pellets is greatly influenced by the characteristics of orifice of the screen. And increase in orifice dimension resulted in increased mean pellet size [20].

\section{CONCLUSION}

This review gives brief idea on pellets and pellatization technique especially emphasizing on Extrusion -Spheronization and Solution/suspension layering. Pelletized dosage forms as one of the most promising and efficient way of novel and multiparticulate drug delivery system. The pellets formation technology has remained much of an art of many years due to the process formulation and therapeutic advantage's over the single unit drug delivery system. A pellet is wide growing technology over the granulation formation technique. It can be concluded that due to their good technological and biopharmaceutical advantages, pelletization has gained an importance in modern pharmaceutical science; and pellets are expected to play a major role in design and fabrication of many novel drug delivery systems in the future.

\section{REFERENCES:-}

[1]Bechgaard H, Nielsen GH. Controlledrelease multiple-units and single-unit doses a literature review. Drug Development and Industrial Pharmacy. 1978 Jan 1;4(1):53-67.

[2] Ahir AA, Mali SS, Hajare AA, Bhagwat DA, Patrekar PV. Pelletization technology: Methods and applications-A review. Research Journal of Pharmacy and Technology. 2015;8(2):131-8.

[3] Patel HP, Patel JK, Patel RR, Patel MP. Pellets: A general overview. International journal of pharma world research. 2010;1(2):1-5.

[4] Jalal IM, Malinowski HJ, Smith WE. Tablet granulations composed of spherical-shaped particles. Journal of pharmaceutical sciences. 1972 Sep;61(9):1466-8.

[5] Eskilson C. Controlled release by microencapsulation. Manufacturing Chemist. 1985;56(3):33-9.

[6] Kandukuri JM, Allenki V, Eaga CM, Keshetty V, Jannu KK. Pelletization techniques for oral drug delivery. International Journal of Pharmaceutical Sciences and drug research. 2009;1(2):63-70.

[7] Hirjau M, Nicoara AC, Hirjau V, Lupuleasa D. Pelletization techniques 
used in pharmaceutical fields. Farma. 2011;4(3):4.

[8] Ghai D. Pelletization: An alternate to granulation. Pharma times. 2011 Jan;43(11):13-5.

[9] Sachan NK, Singh B, Rao KR. Controlled drug delivery through microencapsulation. Malaysian J Pharm Sci. 2006;4(1):65-81.

[10] Manoharan K, Bhaskaran NA, Kumar L. Pellets and techniques of pelletization. Research Journal of Pharmacy and Technology. 2019;12(12):6157-64.

[11] Bathool A, Vishakante GD, Khan MS, Gupta VK. Pelletization as a key tool for oral drug delivery: A review. Journal of Pharmacy Research. 2011 Oct;4(10):3282-6.

[12] Ghebre-Sellassie I. Mechanism of pellet formation and growth. Pharmaceutical pelletization technology. 1989;37:12345.

[13] Kristensen HG, Schaefer T. Granulation: A review on pharmaceutical wetgranulation. Drug development and industrial pharmacy. 1987 Jan 1;13(45):803-72.

[14] Lavanya K, Senthil V, Rathi V. Pelletization technology: a quick review. International Journal of Pharmaceutical
Sciences and Research. 2011 Jun 1;2(6):1337.

[15] Walker GM, Holland CR, Ahmad MM, Craig DQ. Influence of process parameters on fluidised hot-melt granulation and tablet pressing of pharmaceutical powders. Chemical Engineering Science. 2005 Jul 1;60(14):3867-77.

[16] Kovacevic J, Mladenovic A, Djuris J, Ibric S. Evaluation of powder, solution and suspension layering for the preparation of enteric coated pellets. European Journal of Pharmaceutical Sciences. 2016 Mar 31;85:84-93.

[17] Duarte Í, Andrade R, Pinto JF, Temtem M. Green production of cocrystals using a new solvent-free approach by spray congealing. International journal of pharmaceutics. 2016 Jun 15;506(12):68-78.

[18] Sonaglio D, Bataille B, Ortigosa C, Jacob M. Factorial design in the feasibility of producing Microcel MC 101 pellets by extrusion/spheronization. International journal of pharmaceutics. 1995 Feb 28;115(1):53-60.

[19] Vervaet C, Baert L, Remon JP. Extrusion-spheronisation A literature review. International journal of 
pharmaceutics. 1995 Mar 28;116(2):131-

46.

[20] Sahoo GP, Parashar B. Pharmaceutical processing-a review on spheronization technology. Journal of Pharmaceutical Research and Opinion. 2013;3(9):65-8. 